Proyecciones Journal of Mathematics

Vol. 41, $\mathrm{N}^{o}$ 1, pp. 177-195, February 2022.

Universidad Católica del Norte

Antofagasta - Chile

\title{
Soft separation axioms and functions with soft closed graphs
}

\author{
Alias B. Khalaf \\ University of Duhok, Iraq \\ Nehmat K. Ahmed \\ Salahaddin University, Iraq \\ and \\ Qumri H. Hamko \\ Salahaddin University, Iraq
}

Received : February 2020. Accepted : October 2021

\begin{abstract}
Several notions on soft topology are studied and their basic properties are investigated by using the concept of soft open sets and soft closure operators which are derived from the basics of soft set theory established by Molodtsov [7]. In this paper we introduce some soft separation axioms called Soft $R_{0}$ and soft $R_{1}$ in soft topological spaces which are defined over an initial universe with a fixed set of parameters. Many characterizations and properties of these spaces are found. Necessary and sufficient conditions for a soft topological space to be a soft $R_{i}$ for $i=0,1$ space are also presented. Furthermore, the concept of functions with soft closed graph and soft cluster sets are defined. Many results on theses two concepts are proved also it is proved that a function has a soft closed graph if and only if its soft cluster set is degenerate.
\end{abstract}

Subjclass [2010]: Primary: 54A05, 54A10, Secondary: 54C05.

Keywords: soft open set, soft $T_{1}$ space, soft $R_{i}$ space $i=0,1$, soft graph, soft cluster set, soft kernel. 


\section{Introduction}

The study of soft sets and their properties was initiated by Molodtsov [7] in 1999. After his introduction of soft set theory as a common mathematical application in dealing with the vagueness of not well defined objects, Several researchers applying on formal modeling, reasoning and computing. Shabir, M. and Naz, M. [9] in 2011 described a soft topological space and they introduced so many basic notations and gave their properties in detail. Mathematicians gave in several papers different and, many interesting topological concepts such as, soft connectedness defined by Zorlutuna, I. and Cakir, H. [13] in 2015. In the same year Wang p. and He, J. [11], introduced the concept of soft compactness. Separation axioms, have been extended in soft topological spaces and many types of soft separation axioms are introduced. Husain S, and Ahmed B. [4] in 2015 introduced separation axioms by using distinct point in the universal set while in 2018 Bayramov S. and Aras C. G. [2] defined some separation axioms by using distinct soft points.

The aim of this paper, is to introduce and discuss in detail a study of two soft separation axioms which we call them soft $R_{0}$ and $R_{1}$ which are defined over an initial universe with a fixed set of parameters. Also we shed light on the notion of a function with a soft closed graph and the soft cluster set of a function, by using notations of soft open sets and soft points defined in [2].

Throughout the present paper, $X$ will be a nonempty initial universal set and $E$ will be a set of parameters and $A$ be a non-empty subset of $E$. A pair $(F, A)$ is called a soft set over $X$, where $F$ is a mapping $F: A \rightarrow P(X)$. The collection of soft sets $(F, A)$ over a universal set $X$ with the parameter set $A$ is denoted by $S P(X)_{A}$.

\section{Preliminaries}

In this section, we give some definitions and results on soft set theory which will be used in the sequel.

First, we recall the following notions more details about properties of such notions can be found in [1], [2], [3], [4] and [6].

Definition 2.1. For two soft sets $(F, A)$ and $(G, B)$ over a common universe $X$, we say that $(F, A)$ is a soft subset of $(G, B)$, if 
1. $A \subseteq B$ and

2. for all $e \in A, F(e) \subseteq G(e)$

We write $(F, A) \sqsubseteq(G, B)$.

Definition 2.2. The complement of a soft set $(F, A)$ is denoted by $(F, A)^{c}$ or $\tilde{X} \backslash(F, A)$ and is defined by $(F, A)^{c}=\left(F^{c}, A\right)$ where $F^{c}: A \rightarrow P(X)$ is a mapping given by $F^{c}(e)=X \backslash F(e)$, for all $e \in A$.

Definition 2.3. A soft set $(F, A)$ over $X$ is said to be empty soft set denoted by $\tilde{\phi}$ if for all $e \in A, F(e)=\phi$ and $(F, A)$ over $X$ is said to be absolute soft set denoted by $\tilde{A}$ if for all $e \in A, F(e)=X$.

Definition 2.4. The union of two soft sets of $(F, A)$ and $(G, B)$ over the common universe $X$ is the soft set $(H, C)=(F, A) \sqcup(G, B)$, where $C=A \cup B$ and for all $e \in C$,

$$
H(e)=\left\{\begin{array}{llll}
F(e) & : & \text { if } & e \in A-B \\
G(e) & : & \text { if } & e \in B-A \\
F(e) \cup G(e) & : & \text { if } & e \in A \cap B
\end{array}\right.
$$
$e \in A$.

In particular, $(F, A) \sqcup(G, A)=(H, A)$ and $H(e)=F(e) \cup G(e)$ for all

Definition 2.5. The intersection $(H, C)$ of two soft sets $(F, A)$ and $(G, B)$ over a common universe $X$, denoted $(F, A) \sqcap(G, B)$, is defined as $C=A \cap B$, and $H(e)=F(e) \cap G(e)$ for all $e \in C$. $e \in A$.

In particular, $(F, A) \sqcap(G, A)=(H, A)$ and $H(e)=F(e) \cap G(e)$ for all

Definition 2.6. Let $x \in X$, then $(x, E)$ denotes the soft set over $X$ for which $x(e)=\{x\}$, for all $e \in E$.

Let $(F, E)$ be a soft set over $X$ and $x \in X$. We say that $x \in(F, E)$ read as $x$ belongs to the soft set $(F, E)$ whenever $x \in F(e)$ for all $e \in E$.

Definition 2.7. The soft set $(F, E)$ is called a soft point, denoted by $\left(x_{e}, E\right)$ or $x_{e}$, if for the element $e \in E, F(e)=\{x\}$ and $F(e)=\phi$ for all $e \in E \backslash\{e\}$.

We say that $x_{e} \in(G, E)$ if $x \in G(e)$. 
Two soft points $x_{e}$ and $y_{e^{\prime}}$ are distinct if either $x \neq y$ or $e \neq e^{\prime}$. It is clear that $x_{e} \in(x, E)$ always.

Definition 2.8. [9] Let $\tau$ be a collection of soft sets over a universe $X$ with a fixed set $A$ of parameters. Then $\tau \subseteq S P(X)_{A}$ is called a soft topology if,

1. $\tilde{\phi}$ and $\tilde{X}$ belongs to $\tau$.

2. The union of any number of soft sets in $\tau$ belongs to $\tau$.

3. The intersection of any two soft sets in $\tau$ belongs to $\tau$.

The triplet $(X, \tau, A)$ is called a soft topological space over $X$. The members of $\tilde{\tau}$ are called soft open sets in $\tilde{X}$ and complements of them are called soft closed sets in $\tilde{X}$ and they are denoted by $S O(\tilde{X})$ and $S C(\tilde{X})$ respectively. Logical operation on soft set are denoted by usual set theoretical operations with symbol $(\sim)$ above. Soft interior and soft closure are denoted by $\tilde{s} i n t$ and $\tilde{s} c l$ respectively.

Definition 2.9. [9] Let $(X, \tau, A)$ be a soft topological space and let $(G, A)$ be a soft set. Then

1. The soft closure of $(G, A)$ is the soft set

$$
\tilde{s} c l(G, A)=\sqcap\{(K, B) \in S C(\tilde{X}):(G, A) \sqsubseteq(K, B)\}
$$

2. The soft interior of $(G, A)$ is the soft set $\tilde{\operatorname{sint}}(G, A)=\sqcup\{(H, B) \in S O(\tilde{X}):(H, B) \sqsubseteq(G, A)\}$.

Definition 2.10. [3] Let $(X, \tau, A)$ be a soft topological space, $(G, A)$ be a soft set over $\tilde{X}$ and $x_{e} \in \tilde{X}$. Then $(G, A)$ is said to be a soft neighborhood of $x_{e}$ if there exists a soft open set $(H, A)$ such that $x_{e} \in(H, A) \sqsubseteq((G, A)$.

Definition 2.11. [5] Let $S P(X)_{A}$ and $S P(Y)_{B}$ be families of soft sets. Let $u: X \rightarrow Y$ and $p: A \rightarrow B$ be mappings. Then a mapping $f_{p u}$ : $S P(X)_{A} \rightarrow S P(Y)_{B}$ is defined as:

1. Let $(F, A)$ be a soft set in $S P(X)_{A}$. The image of $(F, A)$ under $f_{p u}$, written as $f_{p u}(F, A)=\left(f_{p u}(F), p(A)\right)$, is a soft set in $S P(Y)_{B}$ such that

$$
f_{p u}(F)\left(e^{\prime}\right)=\left\{\begin{array}{llll}
\bigcup_{e \in p^{-1}\left(e^{\prime}\right) \cap A} u(F(e)) & : & \text { if } & p^{-1}\left(e^{\prime}\right) \cap A \neq \phi \\
\phi & : & \text { if } & p^{-1}\left(e^{\prime}\right) \cap A=\phi
\end{array}\right.
$$

for all $e^{\prime} \in B$. 
2. Let $(G, B)$ be a soft set in $S P(Y)_{B}$. Then the inverse image of $(G, B)$ under $f_{p u}$, written as $f_{p u}^{-1}(G, B)=\left(f_{p u}^{-1}(G), p^{-1}(B)\right)$, is a soft set in $S P(X)_{A}$ such that

$$
f_{p u}^{-1}(G)(e)= \begin{cases}u^{-1}(G(p(e))) & : \text { if } p(e) \in B \\ \phi & : \text { otherwise }\end{cases}
$$

for all $e \in A$.

The soft function $f_{p u}$ is called surjective if $p$ and $u$ are surjective and it is called injective if $p$ and $u$ are injective.

Definition 2.12. [13] Let $(X, \tau, A)$ and $(Y, \mu, B)$ be two soft topological spaces. A soft mapping $f_{p u}: \tilde{X} \rightarrow \tilde{Y}$ is called soft continuous if $f_{p u}^{-1}((G, B)) \in \tau$ for all $(G, B) \in \mu$.

Definition 2.13. [12] A soft filter $\mathcal{F}$ converges to a soft point $x_{e} \in \tilde{X}$ in a soft topological space $(X, \tau, A)$, if every soft neighborhood of the soft point $x_{e}$ belongs to the soft filter $\mathcal{F}$. It is denoted by $\mathcal{F} \rightarrow x_{e}$.

Definition 2.14. [11] Let $\mathcal{F}$ be a soft filter in a soft topological space $(X, \tau, A)$, a soft point $x_{e}$ is called soft accumulation point of $\mathcal{F}$, if $x_{e} \in$ $\tilde{s} c l(G, A)$ for every $(G, A) \in \mathcal{F}$.

Theorem 2.15. [11] A soft filter $\mathcal{F}$ converges to a soft point $x_{e}$, then $x_{e}$ is the soft accumulation point of $\mathcal{F}$, if $\mathcal{F}$ is a maximal soft filter and $x_{e}$ is a soft accumulation point of $\mathcal{F}$, then the soft filter $\mathcal{F}$ converges to the soft point $x_{e}$.

Proposition 2.16. [8] Let $f_{p u}: S P(X)_{A} \rightarrow S P(Y)_{B}$ be a soft function. If $\mathcal{F}$ is a soft ultra filter in $\tilde{X}$, then $f_{p u}(\mathcal{F})$ is a soft ultra filter in $\tilde{Y}$.

Definition 2.17. [2] A soft topological space $(X, \tau, A)$ is said to be:

1. Soft $T_{0}$, if for each pair of distinct soft points $x_{e}, y_{e^{\prime}} \in S P(X)_{A}$, there exist soft open sets $(F, A)$ and $(G, A)$ such that either $x_{e} \in(F, A)$ and $y_{e^{\prime}} \notin(F, A)$ or $y_{e^{\prime}} \in(G, A)$ and $x_{e} \notin(G, A)$.

2. Soft $T_{1}$, if for each pair of distinct soft points $x_{e}, y_{e^{\prime}} \in S P(X)_{A}$, there exist two soft open sets $(F, A)$ and $(G, A)$ such that $x_{e} \in(F, A)$ but $y_{e^{\prime}} \notin(F, A)$ and $y_{e^{\prime}} \in(G, A)$ but $x_{e} \notin(G, A)$. 
3. Soft $T_{2}$, if for each pair of distinct soft points $x_{e}, y_{e^{\prime}} \in S P(X)_{A}$, there exist two disjoint soft open sets $(F, A)$ and $(G, A)$ containing $x_{e}$ and $y_{e^{\prime}}$ respectively.

Proposition 2.18. [2]

1. Every soft $T_{2}$-space $\Rightarrow$ soft $T_{1}$-space $\Rightarrow$ soft $T_{0}$-space.

2. A soft topological space $(X, \tau, A)$ is soft $T_{1}$ if and only if each soft point is soft closed.

\section{Soft $R_{0}$ and soft $R_{1}$ Spaces}

In this section we introduce new types of soft separation axioms over the universal set $X$ and a fixed set of parameters by using the soft points defined in [2] called soft $R_{i}$ spaces for $i=0,1$. We obtain several properties and characterizations of these spaces.

Definition 3.1. A soft topological space $(X, \tau, A)$ is called soft $R_{0}$ if for every soft open set $(F, A), \tilde{s} c l\left(\left\{x_{e}\right\}\right) \sqsubseteq(F, A)$ whenever $x_{e} \in(F, A)$.

Definition 3.2. A soft topological space $(X, \tau, A)$ is called soft $R_{1}$ if for $x_{e}, y_{e^{\prime}} \in \tilde{X}$ with $\tilde{s} c l\left(\left\{x_{e}\right\}\right) \neq \tilde{s} c l\left(\left\{y_{e^{\prime}}\right\}\right)$, there exist disjoint soft open sets $(F, A)$ and $(G, A)$ such that $\tilde{s} c l\left(\left\{x_{e}\right\}\right) \sqsubseteq(F, A)$ and $\tilde{s} c l\left(\left\{y_{e^{\prime}}\right\}\right) \sqsubseteq(G, A)$.

Definition 3.3. Let $(X, \tau, A)$ be a soft topological space, then the soft kernel of the soft set $(F, A)$ is defined to be the intersection of all soft open sets containing $(F, A)$ and it is denoted by $\tilde{s} k e r(F, A)$ that is $\tilde{s} k e r(F, A)=$ $\sqcap\{(G, A) \in S O(\tilde{X}):(F, A) \sqsubseteq(G, A)\}$.

Lemma 3.4. For any two soft points $x_{e}$ and $y_{e^{\prime}}$ in a soft topological space $(X, \tau, A)$, we have $y_{e^{\prime}} \in \tilde{s} k e r\left(\left\{x_{e}\right\}\right)$ if and only if $x_{e} \in \tilde{s} c l\left(\left\{y_{e^{\prime}}\right\}\right)$.

Proof. Suppose thaty $y_{e^{\prime}} \notin \tilde{s} k e r\left(\left\{x_{e}\right\}\right)$, then there exists a soft open sets $(F, A)$ containing $x_{e}$ such that $y_{e^{\prime}} \notin(F, A)$. Therefore, we have $x_{e} \notin$ $\tilde{s} c l\left(\left\{y_{e^{\prime}}\right\}\right)$. The proof of the converse part is similar.

In the following theorem, we give characterizations of soft $R_{0}$ spaces.

Theorem 3.5. Let $(X, \tau, A)$ be a soft topological space, then the following properties are equivalent:

1. $(X, \tau, A)$ is $R_{0}$, 
2. For any $(K, A) \in S C(\tilde{X})$ and $x_{e} \notin(K, A)$, there exists $(F, A) \in$ $S C(\tilde{X})$ such that $(K, A) \sqsubseteq(F, A)$ and $x_{e} \notin(F, A)$,

3. For any $(K, A) \in S C(\tilde{X})$ and $x_{e} \notin(K, A)$, implies that $(K, A) \sqcap$ $\tilde{s} c l\left(\left\{x_{e}\right\}\right)=\tilde{\phi}$,

4. For any distinct soft points $x_{e}, y_{e^{\prime}} \in \tilde{X}$, either $\tilde{s} c l\left(\left\{x_{e}\right\}\right)=\tilde{s} c l\left(\left\{y_{e^{\prime}}\right\}\right)$ or $\tilde{s} c l\left(\left\{x_{e}\right\}\right) \sqcap \tilde{s} c l\left(\left\{y_{e^{\prime}}\right\}\right)=\tilde{\phi}$.

Proof. $\quad(1) \Rightarrow(2)$. Let $(K, A) \in S C(\tilde{X})$ and $x_{e} \notin(K, A)$. Then by (1), $\tilde{s} c l\left(\left\{x_{e}\right\}\right) \sqsubseteq \tilde{X} \backslash(K, A)$. Let $(F, A)=\tilde{X} \backslash(K, A)$, then $(F, A) \in S O(\tilde{X})$, $(K, A) \sqsubseteq(F, A)$ and $x_{e} \notin(F, A)$.

$(2) \Rightarrow(3)$. Let $(K, A) \in S C(\tilde{X})$ and $x_{e} \notin(K, A)$. Then there exists $(F, A) \in$ $S O(\tilde{X})$ such that $(K, A) \sqsubseteq(F, A)$ and $x_{e} \notin(F, A)$. Hence, by $(2),(F, A) \sqcap$ $\tilde{s} c l\left(\left\{x_{e}\right\}\right)=\tilde{\phi}$, this implies that $(K, A) \sqcap \tilde{s} c l\left(\left\{x_{e}\right\}\right)=\tilde{\phi}$.

$(3) \Rightarrow(4)$. Let $x_{e}$ and $y_{e^{\prime}}$ be two distinct soft points of $\tilde{X}$. Suppose that $\tilde{s} c l\left(\left\{x_{e}\right\}\right) \neq \tilde{s} c l\left(\left\{y_{e^{\prime}}\right\}\right)$, then there exists a soft point $z_{c}$ such that $z_{c} \in$ $\tilde{s} c l\left(\left\{x_{e}\right\}\right)$ and $z_{c} \notin \tilde{s} c l\left(\left\{y_{e^{\prime}}\right\}\right)$ [ or $z_{c} \in \tilde{s} c l\left(\left\{y_{e^{\prime}}\right\}\right)$ and $\left.z_{c} \notin \tilde{s} c l\left(\left\{x_{e}\right\}\right)\right]$ and there exists $(F, A) \in S O(\tilde{X})$ such that $y_{e^{\prime}} \notin(F, A)$ and $z_{c} \in(F, A)$, so $x_{e} \in(F, A)$. Therefore, we get $x_{e} \notin \tilde{s} c l\left(\left\{y_{e^{\prime}}\right\}\right)$, then by (3), we obtain $\tilde{s} c l\left(\left\{x_{e}\right\}\right) \sqcap \tilde{s} c l\left(\left\{y_{e^{\prime}}\right\}\right)=\tilde{\phi}$.

$(4) \Rightarrow(1)$. Let $(F, A) \in S O(\tilde{X})$ and $x_{e} \in(F, A)$, for each $y_{e^{\prime}} \notin(F, A)$. Hence, $x_{e} \neq y_{e^{\prime}}$ and $x_{e} \notin \tilde{s} c l\left(\left\{y_{e^{\prime}}\right\}\right)$, this shows that $\tilde{s} c l\left(\left\{x_{e}\right\}\right) \neq \tilde{s} c l\left(\left\{y_{e^{\prime}}\right\}\right)$. By (4), we have $\tilde{s} c l\left(\left\{x_{e}\right\}\right) \sqcap \tilde{s} c l\left(\left\{y_{e^{\prime}}\right\}\right)=\tilde{\phi}$, for each $y_{e^{\prime}} \in \tilde{X} \backslash(F, A)$. On the other hand, since $(F, A) \in S O(\tilde{X})$ and $y_{e^{\prime}} \in \tilde{X} \backslash(F, A)$, we have $\tilde{s} c l\left(\left\{y_{e^{\prime}}\right\}\right) \sqsubseteq$ $\tilde{X} \backslash(F, A)$. Hence $\tilde{X} \backslash(F, A)=\sqcup \tilde{s} c l\left(\left\{y_{e^{\prime}}\right\}\right)$ where $y_{e^{\prime}} \in \tilde{X} \backslash(F, A)$. Therefore we obtain that $\tilde{X} \backslash(F, A) \sqcap \tilde{s} c l\left(\left\{y_{e^{\prime}}\right\}\right)=\tilde{\phi}$ and $\tilde{s} c l\left(\left\{x_{e}\right\}\right) \sqsubseteq(F, A)$. This shows that $(X, \tau, A)$ is $R_{0}$.

Lemma 3.6. Let $x_{e}$ and $y_{e^{\prime}}$ be any distinct soft points in a soft topological space $(X, \tau, A)$. Then $\tilde{s} k e r\left(\left\{x_{e}\right\}\right) \neq \tilde{s} k e r\left(\left\{y_{e^{\prime}}\right\}\right)$ if and only if $\tilde{s} c l\left(\left\{x_{e}\right\}\right) \neq$ $\tilde{s} c l\left(\left\{y_{e^{\prime}}\right\}\right)$.

Proof. Suppose that $\tilde{s} k e r\left(\left\{x_{e}\right\}\right) \neq \tilde{s} k e r\left(\left\{y_{e^{\prime}}\right\}\right)$. Then there exists a soft point $z_{c} \in \tilde{X}$ such that $z_{c} \in \tilde{s} k e r\left(\left\{x_{e}\right\}\right)$ and $z_{c} \notin \tilde{s} k e r\left(\left\{y_{e^{\prime}}\right\}\right)$. Since $z_{c} \in \tilde{s} k e r\left(\left\{x_{e}\right\}\right)$ so $\left\{x_{e}\right\} \sqcap \tilde{s} c l\left(\left\{z_{c}\right\}\right) \neq \tilde{\phi}$. This implies that $x_{e} \in \tilde{s} c l\left(\left\{z_{c}\right\}\right)$ and since $z_{c} \notin \tilde{s} c l\left(\left\{y_{e^{\prime}}\right\}\right)$ we have $\left\{y_{e^{\prime}}\right\} \sqcap \tilde{s} c l\left(\left\{z_{c}\right\}\right)=\tilde{\phi}$. Since $x_{e} \in \tilde{s} c l\left(\left\{z_{c}\right\}\right)$, so $\tilde{s} c l\left(\left\{x_{e}\right\}\right) \sqsubseteq \tilde{s} c l\left(\left\{z_{c}\right\}\right)$ and hence $\left\{y_{e^{\prime}}\right\} \sqcap \tilde{s} c l\left(\left\{x_{e}\right\}\right)=\tilde{\phi}$. Therefore, $\tilde{s} c l\left(\left\{x_{e}\right\}\right) \neq \tilde{s} c l\left(\left\{y_{e^{\prime}}\right\}\right)$.

Conversely, Suppose that $\tilde{s} c l\left(\left\{x_{e}\right\}\right) \neq \tilde{s} c l\left(\left\{y_{e^{\prime}}\right\}\right)$. Then there exists a soft 
point $z_{c} \in \tilde{X}$ such that $z_{c} \in \tilde{s} c l\left(\left\{x_{e}\right\}\right)$ and $z_{c} \notin \tilde{s} c l\left(\left\{y_{e^{\prime}}\right\}\right)$. Hence, there exists a soft open set $(F, A)$ containing $z_{c}$ (and hence $x_{e}$ ) but not $y_{e^{\prime}}$, that is $y_{e^{\prime}} \notin \tilde{s} k e r\left(\left\{x_{e}\right\}\right)$. Therefore, $\tilde{s} k e r\left(\left\{x_{e}\right\}\right) \neq \tilde{s} k e r\left(\left\{y_{e^{\prime}}\right\}\right)$.

Theorem 3.7. A soft topological space $(X, \tau, A)$ is soft $R_{0}$ if and only if for any two distinct soft points $x_{e}, y_{e^{\prime}} \in \tilde{X}, \tilde{s} k e r\left(\left\{x_{e}\right\}\right) \neq \tilde{s} k e r\left(\left\{y_{e^{\prime}}\right\}\right)$ implies $\tilde{s} k e r\left(\left\{x_{e}\right\}\right) \sqcap \tilde{s} k e r\left(\left\{y_{e^{\prime}}\right\}\right)=\tilde{\phi}$.

Proof. Necessity, suppose that $(X, \tau, A)$ is soft $R_{0}$. Thus by Lemma 3.6, for any distinct soft points $x_{e}, y_{e^{\prime}} \in \tilde{X}$, if $\tilde{s} k e r\left(\left\{x_{e}\right\}\right) \neq \tilde{s} k e r\left(\left\{y_{e^{\prime}}\right\}\right)$, then $\tilde{s} c l\left(\left\{x_{e}\right\}\right) \neq \tilde{s} c l\left(\left\{y_{e^{\prime}}\right\}\right)$. Assume that $z_{c} \in \tilde{s} k e r\left(\left\{x_{e}\right\}\right) \sqcap \tilde{s} k e r\left(\left\{y_{e^{\prime}}\right\}\right)$. Since $z_{c} \in \tilde{s} k e r\left(\left\{x_{e}\right\}\right)$ and by Lemma 3.4, it follows that $x_{e} \in \tilde{s} c l\left(\left\{z_{c}\right\}\right)$. Since $x_{e} \in \tilde{s} c l\left(\left\{x_{e}\right\}\right)$, by Theorem $3.5 \tilde{s} c l\left(\left\{x_{e}\right\}\right)=\tilde{s} c l\left(\left\{y_{e^{\prime}}\right\}\right)$. Similarly we have $\tilde{s} c l\left(\left\{x_{e}\right\}\right)=\tilde{s} c l\left(\left\{z_{c}\right\}\right)=\tilde{s} c l\left(\left\{y_{e^{\prime}}\right\}\right)$, which is a contradiction. Therefore $\tilde{s} k e r\left(\left\{x_{e}\right\}\right) \sqcap \tilde{s} k \operatorname{er}\left(\left\{y_{e^{\prime}}\right\}\right)=\tilde{\phi}$.

Sufficiency, let $(X, \tau, A)$ be a soft topological space such that for any distinct soft points $x_{e}, y_{e^{\prime}} \in \tilde{X}, \tilde{s} k e r\left(\left\{x_{e}\right\}\right) \neq \tilde{s} k e r\left(\left\{y_{e^{\prime}}\right\}\right)$ implies that $\tilde{s} k \operatorname{er}\left(\left\{x_{e}\right\}\right) \sqcap \tilde{s} k \operatorname{er}\left(\left\{y_{e^{\prime}}\right\}\right)=\tilde{\phi}$. If $\tilde{s} c l\left(\left\{x_{e}\right\}\right) \neq \tilde{s} c l\left(\left\{y_{e^{\prime}}\right\}\right)$, then by Lemma 3.6, šser $\left(\left\{x_{e}\right\}\right) \neq \tilde{s} k \operatorname{er}\left(\left\{y_{e^{\prime}}\right\}\right)$. Therefore $\tilde{s} k \operatorname{er}\left(\left\{x_{e}\right\}\right) \sqcap \tilde{s} k \operatorname{er}\left(\left\{y_{e^{\prime}}\right\}\right)=\tilde{\phi}$. Now if $z_{c} \in \tilde{s} c l\left(\left\{x_{e}\right\}\right) \sqcap \tilde{s} c l\left(\left\{y_{e^{\prime}}\right\}\right)$, then $z_{c} \in \tilde{s} c l\left(\left\{x_{e}\right\}\right)$ implies that $x_{e} \in$ $\tilde{s} k e r\left(\left\{z_{c}\right\}\right)$ so, $\tilde{s} k e r\left(\left\{x_{e}\right\}\right) \sqcap \tilde{s} k e r\left(\left\{z_{c}\right\}\right) \neq \tilde{\phi}$ and by the same way we obtain that $\tilde{s} k e r\left(\left\{y_{e^{\prime}}\right\}\right) \sqcap \tilde{s} k \operatorname{er}\left(\left\{z_{c}\right\}\right) \neq \tilde{\phi}$. By hypothesis, we have, $\tilde{s} k e r\left(\left\{x_{e}\right\}\right)=$ $\tilde{s} k e r\left(\left\{z_{c}\right\}\right)=\tilde{s} k \operatorname{er}\left(\left\{y_{e^{\prime}}\right\}\right)$, which is contradiction so, $\tilde{s} c l\left(\left\{x_{e}\right\}\right) \sqcap \tilde{s} c l\left(\left\{y_{e^{\prime}}\right\}\right)=$ $\tilde{\phi}$. Therefore, by Theorem 3.5, $(X, \tau, A)$ is soft $R_{0}$.

Theorem 3.8. Let $(X, \tau, A)$ be a soft topological space. Then the following statements are equivalent:

1. $(X, \tau, A)$ is soft $R_{0}$,

2. For any non-empty soft set $(F, A),(G, A) \in S O(\tilde{X})$ with $(F, A) \sqcap$ $(G, A) \neq \tilde{\phi}$, there exists $(K, A) \in S C(\tilde{X})$ such that $(F, A) \sqcap(K, A) \neq$ $\tilde{\phi}$, and $(K, A) \sqsubseteq(G, A)$.

3. For any $(G, A) \in S O(\tilde{X}),(G, A)=\sqcup\{(K, A) \in S C(\tilde{X}):(K, A) \sqsubseteq$ $(G, A)\}$,

4. For any $(K, A) \in S C(\tilde{X}),(K, A)=\sqcap\{(G, A) \in S O(\tilde{X}):(K, A) \sqsubseteq$ $(G, A)\}$,

5. For any $x_{e} \in \tilde{X}, \tilde{s} c l\left(\left\{x_{e}\right\}\right) \sqsubseteq \tilde{s} k e r\left(\left\{x_{e}\right\}\right)$. 
Proof. $\quad(1) \Rightarrow(2)$. Let $(F, A)$ be a non empty subset of $\tilde{X}$ and $(G, A) \in$ $S O(\tilde{X})$ such that $(F, A) \sqcap(G, A) \neq \tilde{\phi}$. Let $x_{e} \in(F, A) \sqcap(G, A)$. Since $x_{e} \in(G, A) \in S O(\tilde{X})$, so by $(1)$, we have $\tilde{s} c l\left(\left\{x_{e}\right\}\right) \sqsubseteq(G, A)$. Set $(K, A)=\tilde{s} c l\left(\left\{x_{e}\right\}\right)$, then $(K, A) \in S C(\tilde{X})$ such that $(K, \bar{A}) \sqsubseteq(G, A)$ and $(F, A) \sqcap(K, A) \neq \tilde{\phi}$.

$(2) \Rightarrow(3)$. Let $(G, A) \in S O(\tilde{X})$. Then $\sqcup\{(K, A) \in S C(\tilde{X}):(K, A) \sqsubseteq$ $(G, A)\} \sqsubseteq(G, A)$. Now let $x_{e}$ be any soft point of $(G, A)$. By $(2)$, there exists $(K, A) \in S C(\tilde{X})$, such that $x_{e} \in(K, A)$ and $(K, A) \sqsubseteq(G, A)$. Therefore, we have $x_{e} \in(K, A) \sqsubseteq \sqcup\{(K, A) \in S C(\tilde{X}):(K, A) \sqsubseteq(G, A)\}$. Hence $(G, A)=\sqcup\{(K, A) \in S C(\tilde{X}):(K, A) \sqsubseteq(G, A)\}$.

$(3) \Rightarrow(4)$. Obvious.

$(4) \Rightarrow(5)$. Let $x_{e}$ be any soft point of $\tilde{X}$ and $y_{e^{\prime}} \notin \tilde{s} k e r\left(\left\{x_{e}\right\}\right)$. So there exists $(H, A) \in S O(\tilde{X})$ such that $x_{e} \in(H, A)$ and $y_{e^{\prime}} \notin(H, A)$. Hence $\tilde{s} c l\left(\left\{y_{e^{\prime}}\right\}\right) \sqcap(H, A)=\tilde{\phi}$. By $(4)$, we have $\left[\sqcap\left\{(G, A) \in S O(\tilde{X}): \tilde{s} c l\left(\left\{y_{e^{\prime}}\right\}\right) \sqsubseteq\right.\right.$ $(G, A)\}] \sqcap(H, A)=\tilde{\phi}$, so $x_{e} \notin(G, A)$ and $\tilde{s} c l\left(\left\{y_{e^{\prime}}\right\}\right) \sqsubseteq(G, A)$. Therefore, $\tilde{s} c l\left(\left\{x_{e}\right\}\right) \sqcap(G, A)=\tilde{\phi}$ and hence $y_{e^{\prime}} \notin \tilde{s} c l\left(\left\{x_{e}\right\}\right)$. Consequently we obtain that $\tilde{s} c l\left(\left\{x_{e}\right\}\right) \sqsubseteq \tilde{s} k e r\left(\left\{x_{e}\right\}\right)$.

$(5) \Rightarrow(1)$. Let $(G, A) \in S O(\tilde{X})$ and $x_{e} \in(G, A)$, let $y_{e^{\prime}} \in \tilde{s} k e r\left(\left\{x_{e}\right\}\right)$. Then $x_{e} \in \tilde{s} c l\left(\left\{y_{e^{\prime}}\right\}\right)$ and $y_{e^{\prime}} \in(G, A)$ this implies that $\tilde{s} k e r\left(\left\{x_{e}\right\}\right) \sqsubseteq(G, A)$. Therefore, we obtain $\tilde{s} c l\left(\left\{x_{e}\right\}\right) \sqsubseteq \tilde{s} k e r\left(\left\{x_{e}\right\}\right) \sqsubseteq(G, A)$. This shows that $(X, \tau, A)$ is soft $R_{0}$.

Theorem 3.9. A soft topological space $(X, \tau, A)$ is soft $R_{0}$ if and only if $\tilde{s} c l\left(\left\{x_{e}\right\}\right)=\tilde{s} k e r\left(\left\{x_{e}\right\}\right)$, for all $x_{e} \in \tilde{X}$.

Proof. Suppose that $(X, \tau, A)$ is a soft $R_{0}$ space. By Theorem 3.8(5), $\tilde{s} c l\left(\left\{x_{e}\right\}\right) \sqsubseteq \tilde{s} k e r\left(\left\{x_{e}\right\}\right)$ for all $x_{e} \in \tilde{X}$. Let $y_{e^{\prime}} \in \tilde{s} k e r\left(\left\{x_{e}\right\}\right)$, then $x_{e} \in$ $\tilde{s} c l\left(\left\{y_{e^{\prime}}\right\}\right)$ and by Theorem 3.8, $\tilde{s} c l\left(\left\{x_{e}\right\}\right)=\tilde{s} c l\left(\left\{y_{e^{\prime}}\right\}\right)$. Therefore, $y_{e^{\prime}} \in$ $\tilde{s} c l\left(\left\{x_{e}\right\}\right)$ and hence $\tilde{s} k e r\left(\left\{x_{e}\right\}\right) \sqsubseteq \tilde{s} c l\left(\left\{x_{e}\right\}\right)$. This shows that $\tilde{s} c l\left(\left\{x_{e}\right\}\right)=$ $\tilde{s} k e r\left(\left\{x_{e}\right\}\right)$, for all $x_{e} \in \tilde{X}$.

The converse part follows from Theorem 3.8.

Theorem 3.10. For a soft topological space $(X, \tau, A)$, the following statement are equivalent :

1. $(X, \tau, A)$ is a soft $R_{0}$ space,

2. $x_{e} \in \tilde{s} c l\left(\left\{y_{e^{\prime}}\right\}\right)$ if and only if $y_{e^{\prime}} \in \tilde{s} c l\left(\left\{x_{e}\right\}\right)$ for any two distinct soft points $x_{e}, y_{e^{\prime}} \in \tilde{X}$. 
Proof. $\quad(1) \Rightarrow(2)$. Assume that $(X, \tau, A)$ is a soft $R_{0}$ space. Let $x_{e} \in \tilde{s} c l\left(\left\{y_{e^{\prime}}\right\}\right)$ and $(H, A)$ be any soft open set containing $y_{e^{\prime}}$, so by definition, $\tilde{s} c l\left(\left\{y_{e^{\prime}}\right\}\right) \sqsubseteq(H, A)$, hence $x_{e} \in(H, A)$. Therefore, every soft open set containing $y_{e^{\prime}}$ contains $x_{e}$, so $y_{e^{\prime}} \in \tilde{s} c l\left(\left\{x_{e}\right\}\right)$.

$(2) \Rightarrow(1)$. Let $(G, A)$ be any soft open set containing $x_{e}$, if $y_{e^{\prime}} \notin(G, A)$, then $x_{e} \notin \tilde{s} c l\left(\left\{y_{e^{\prime}}\right\}\right)$ and By $(2)$, we have $y_{e^{\prime}} \notin \tilde{s} c l\left(\left\{x_{e}\right\}\right)$. This implies that $\tilde{s} c l\left(\left\{x_{e}\right\}\right) \sqsubseteq(G, A)$, hence $(X, \tau, A)$ is a soft $R_{0}$ space.

The following corollary follows from Theorem 3.9 and Theorem 3.10.

Corollary 3.11. A soft topological space $(X, \tau, A)$ is a soft $R_{0}$ space if and only if $\tilde{s} k e r\left(\left\{x_{e}\right\}\right) \neq \tilde{s} k e r\left(\left\{y_{e^{\prime}}\right\}\right)$ for all distinct soft points $x_{e}, y_{e^{\prime}} \in \tilde{X}$.

Lemma 3.12. Let $(X, \tau, A)$ be a soft topological space and $(F, A) \sqsubseteq \tilde{X}$. Then $\tilde{s} k e r(F, A)=\left\{x_{e} \in \tilde{X}: \tilde{s} c l\left(\left\{x_{e}\right\}\right) \sqcap(F, A) \neq \tilde{\phi}\right\}$.

Proof. $\quad$ Let $x_{e} \in \tilde{s} k e r(F, A)$ and $\tilde{s} c l\left(\left\{x_{e}\right\}\right) \sqcap(F, A)=\tilde{\phi}$. So we have $x_{e} \notin \tilde{X} \backslash \tilde{s} c l\left(\left\{x_{e}\right\}\right)$ which is a soft open set containing $(F, A)$. This is impossible, because $x_{e} \in \tilde{s} k e r(F, A)$. Therefore, $\tilde{s} c l\left(\left\{x_{e}\right\}\right) \sqcap(F, A) \neq \tilde{\phi}$. On the other hand, if $\tilde{s} c l\left(\left\{x_{e}\right\}\right) \sqcap(F, A) \neq \tilde{\phi}$ and $x_{e} \notin \tilde{s} k e r(F, A)$. Then there exists a soft open set $(H, A)$ containing $(F, A)$ and $x_{e} \notin(H, A)$. Let $y_{e^{\prime}} \in \tilde{s} c l\left(\left\{x_{e}\right\}\right) \sqcap(F, A)$, so $(H, A)$ is a soft neighborhood of $y_{e^{\prime}}$ in which $x_{e} \notin(H, A)$, which is a contradiction, so $x_{e} \in \tilde{s} k e r(F, A)$.

Theorem 3.13. For a soft topological space $(X, \tau, A)$, The following statement are equivalent:

1. $(X, \tau, A)$ is a soft $R_{0}$,

2. If $(K, A)$ is soft closed, then $(K, A)=\tilde{s} k e r(K, A)$,

3. If $(K, A)$ is soft closed and $x_{e} \in(K, A)$, then $\tilde{s} k e r\left(\left\{x_{e}\right\}\right) \sqsubseteq(K, A)$,

4. If $x_{e} \in \tilde{X}$, then $\tilde{s} k e r\left(\left\{x_{e}\right\}\right) \sqsubseteq \tilde{s} c l\left(\left\{x_{e}\right\}\right)$.

Proof. $\quad(1) \Rightarrow(2)$. Let $(K, A)$ be a soft closed set and $x_{e} \notin(K, A)$. Then $\tilde{X} \backslash(K, A)$ is soft open set containing $x_{e}$. Since $\tilde{X}$ is a soft $R_{0}$ space, so $\tilde{s} c l\left(\left\{x_{e}\right\}\right) \sqsubseteq \tilde{X} \backslash(K, A)$, thus $\tilde{s} c l\left(\left\{x_{e}\right\}\right) \sqcap(K, A)=\tilde{\phi}$, by Lemma 3.12, $x_{e} \notin$ $\tilde{s} k e r(K, A)$. Therefore, $\tilde{s} k e r\left(\left\{x_{e}\right\}\right) \sqsubseteq(K, A)$, hence $(K, A)=\tilde{s} k e r(K, A)$. $(2) \Rightarrow(3)$. In general $(F, A) \sqsubseteq(G, A)$ implies that $\tilde{s} k e r(F, A) \sqsubseteq \tilde{s} k e r(G, A)$. Therefore, from (2), it follows that $\tilde{s} k e r\left(\left\{x_{e}\right\}\right) \sqsubseteq(K, A)$. 
$(3) \Rightarrow(4)$. Since $x_{e} \in \tilde{s} c l\left(\left\{x_{e}\right\}\right)$ and $\tilde{s} c l\left(\left\{x_{e}\right\}\right)$ is soft closed, so by (3), we get that $\tilde{s} k e r\left(\left\{x_{e}\right\}\right) \sqsubseteq \tilde{s} c l\left(\left\{x_{e}\right\}\right)$.

$(4) \Rightarrow(1)$. Let $x_{e} \in \tilde{s} c l\left(\left\{y_{e^{\prime}}\right\}\right)$, then by Lemma 3.4, $y_{e^{\prime}} \in \tilde{s} k e r\left(\left\{x_{e}\right\}\right)$. Since $x_{e} \in \tilde{s} c l\left(\left\{x_{e}\right\}\right)$ and $\tilde{s} c l\left(\left\{x_{e}\right\}\right)$ is soft closed, by (4) we obtain $y_{e^{\prime}} \in$ $\tilde{s} k e r\left(\left\{x_{e}\right\}\right) \sqsubseteq \tilde{s} c l\left(\left\{x_{e}\right\}\right)$. Therefore, $x_{e} \in \tilde{s} c l\left(\left\{y_{e^{\prime}}\right\}\right)$ implies that $y_{e^{\prime}} \in$ $\tilde{s} c l\left(\left\{x_{e}\right\}\right)$. Similarly, if $y_{e^{\prime}} \in \tilde{s} c l\left(\left\{x_{e}\right\}\right)$, we get $x_{e} \in \tilde{s} c l\left(\left\{y_{e^{\prime}}\right\}\right)$, so by Theorem $3.10,(X, \tau, A)$ is a soft $R_{0}$.

Proposition 3.14. If a soft topological space $(X, \tau, A)$ is a soft $R_{1}$ space, then it is soft $R_{0}$.

Proof. Suppose that $(X, \tau, A)$ is soft $R_{1}$. Let $(H, A)$ be any soft open set containing a soft point $x_{e}$. Then for each $y_{e^{\prime}} \in \tilde{X} \backslash(H, A), \tilde{s} c l\left(\left\{x_{e}\right\}\right) \neq$ $\tilde{s} c l\left(\left\{y_{e^{\prime}}\right\}\right)$. Since $(X, \tau, A)$ is soft $R_{1}$, there exist two disjoint soft open sets $(K, A)$ and $(G, A)$ such that $\tilde{s} c l\left(\left\{x_{e}\right\}\right) \sqsubseteq(K, A)$ and $\tilde{s} c l\left(\left\{y_{e^{\prime}}\right\}\right) \sqsubseteq(G, A)$. Let $(F, A)=\sqcup\left\{(G, A): y_{e^{\prime}} \in \tilde{X} \backslash(H, A)\right\}$, then $\tilde{X} \backslash(H, A) \sqsubseteq(F, A)$, $x_{e} \notin(F, A)$ and $(F, A)$ is a soft open set. Therefore, $\tilde{s} k e r\left(\left\{x_{e}\right\}\right) \sqsubseteq \tilde{X} \backslash$ $(F, A) \sqsubseteq(H, A)$. Hence, $(X, \tau, A)$ is soft $R_{0}$ space.

Proposition 3.15. If a soft topological space $(X, \tau, A)$ is a soft $T_{1}$ space, then it is soft $R_{0}$.

Proof. The proof is obvious since in a soft $T_{1}$ space, every soft point is soft closed.

The following examples shows that the converses of Proposition 3.14 and Proposition 3.15 are not true in general.

Example 3.16. Let $X$ be any infinite set, $A=\left\{e_{1}, e_{2}\right\}$ and $\tau$ a topology consists of $\tilde{\phi}, \tilde{X}$ and all soft sets $(F, A)$, where $(F, A)$ is defined as: $F\left(e_{1}\right)=$ $G$ where $G$ is a subset of $X$ and $X \backslash G$ is finite and $F\left(e_{2}\right)=\phi$.

Then $(X, \tau, A)$ is a soft topological space over $X$. It can be easily shown that this space is soft $R_{0}$ and not soft $R_{1}$.

Example 3.17. Let $X=\left\{x_{1}, x_{2}\right\}, A=\left\{e_{1}, e_{2}\right\}$ and $\tau=\left\{\tilde{\phi}, \tilde{X},\left(F_{1}, A\right),\left(F_{2}, A\right)\right\}$, where $\left(F_{1}, A\right)=\left\{\left(e_{1},\left\{x_{2}\right\}\right),\left(e_{2},\left\{x_{1}\right\}\right)\right\}$, $\left(F_{2}, A\right)=\left\{\left(e_{1},\left\{x_{1}\right\}\right),\left(e_{2},\left\{x_{2}\right\}\right)\right\}$. Then $(X, \tau, A)$ is a soft topological space over $X$. This space is both soft $R_{0}$ and soft $R_{1}$ but it is not soft $T_{1}$.

Proposition 3.18. A soft topological space $(X, \tau, A)$ is soft $T_{1}$ if and only if it is both soft $T_{0}$ and soft $R_{0}$. 
Proof. Necessity, follows from Proposition 3.15 and the fact that every soft $T_{1}$ is soft $T_{0}$.

Sufficiency, assume that $(X, \tau, A)$ is both soft $T_{0}$ and soft $R_{0}$ space. Let $x_{e}, y_{e^{\prime}} \in \tilde{X}$ be any pair of distinct soft points. Since $(X, \tau, A)$ is both soft $T_{0}$, there exists a soft open set $(H, A)$ which contains one of the points but not the other. Suppose that $x_{e} \in(H, A)$ and $y_{e^{\prime}} \notin(H, A)$. Since $\tilde{X}$ is soft $R_{0}$, then $\tilde{s} c l\left(\left\{x_{e}\right\}\right) \sqsubseteq(H, A)$. As $y_{e^{\prime}} \notin(H, A)$ implies $y_{e^{\prime}} \notin \tilde{s} c l\left(\left\{x_{e}\right\}\right)$. Hence $y_{e^{\prime}} \in(G, A)=\tilde{X} \backslash \tilde{s} c l\left(\left\{x_{e}\right\}\right)$ and it is clear that $x_{e} \notin(G, A)$, this implies that there exist soft open sets $(G, A)$ and $(H, A)$ containing $x_{e}$ and $y_{e^{\prime}}$ respectively such that $x_{e} \notin(G, A)$ and $y_{e^{\prime}} \notin(H, A)$. Therefore, $(X, \tau, A)$ is soft $T_{1}$.

Theorem 3.19. A soft topological space $(X, \tau, A)$ is soft $R_{0}$ if and only if for every soft closed set $(K, A)$ and $x_{e} \notin(K, A)$, there exists a soft open set $(G, A)$ such that $x_{e} \notin(G, A)$ and $(K, A) \sqsubseteq(G, A)$.

Proof. Let $(X, \tau, A)$ be soft $R_{0}, x_{e} \in \tilde{X}$ and $(K, A)$ be soft closed subset such that $x_{e} \notin(K, A)$. Then $\tilde{X} \backslash(K, A)$ is a soft open set containing $x_{e}$. Hence, $\tilde{s} c l\left(\left\{x_{e}\right\}\right) \sqsubseteq \tilde{X} \backslash(K, A)$ and then $(K, A) \sqsubseteq \tilde{X} \backslash \tilde{s} c l\left(\left\{x_{e}\right\}\right)$. Now let $(G, A)=\tilde{X} \backslash \tilde{s} c l\left(\left\{x_{e}\right\}\right)$, then $(G, A)$ is a soft open set does not contains $x_{e}$ and $(K, A) \sqsubseteq(G, A)$.

Conversely: Let $x_{e} \in(G, A)$ where $(G, A)$ is a soft open set in $\tilde{X}$. Then $\tilde{X} \backslash(G, A)$ is a soft closed set and $x_{e} \notin(G, A)$ implies by hypothesis, that there exists a soft open set $(H, A)$ such that $x_{e} \notin(H, A)$ and $\tilde{X} \backslash(G, A) \sqsubseteq$ $(H, A)$. Now $\tilde{X} \backslash(H, A) \sqsubseteq(G, A)$ and $x_{e} \in \tilde{X} \backslash(H, A)$, but $\tilde{X} \backslash(H, A)$ is soft closed, hence $\tilde{s} c l\left(\left\{x_{e}\right\}\right) \sqsubseteq \tilde{X} \backslash(H, A) \sqsubseteq(G, A)$ this implies that $(X, \tau, A)$ is soft $R_{0}$.

Theorem 3.20. A soft topological space $(X, \tau, A)$ is soft $T_{2}$ if and only if it is both soft $T_{0}$ and soft $R_{1}$.

Proof. Let $\tilde{X}$ be soft $T_{2}$, then from Proposition 2.18(1), it is soft $T_{0}$ and to show $\tilde{X}$ is a soft $R_{1}$ space, let $x_{e}, y_{e^{\prime}} \in \tilde{X}$ such that $\tilde{s} c l\left(\left\{x_{e}\right\}\right) \neq \tilde{s} c l\left(\left\{y_{e^{\prime}}\right\}\right)$ and since $\tilde{X}$ is soft $T_{1}$ space, so by Proposition 2.18(2), every singleton set in $\tilde{X}$ is soft closed, that is $\tilde{s} c l\left(\left\{x_{e}\right\}\right)=\left\{x_{e}\right\}$ and $\tilde{s} c l\left(\left\{y_{e^{\prime}}\right\}\right)=\left\{y_{e^{\prime}}\right\}$ and since $\tilde{X}$ is a soft $T_{2}$ space so there exist two disjoint soft open sets $(G, A)$ and $(H, A)$ such that $\left\{x_{e}\right\}=\tilde{s} c l\left(\left\{x_{e}\right\}\right) \sqsubseteq(G, A)$ and $\left\{y_{e^{\prime}}\right\}=\tilde{s} c l\left(\left\{y_{e^{\prime}}\right\}\right) \sqsubseteq(H, A)$. Thus $\tilde{X}$ is soft $R_{1}$ space.

Conversely, let $\tilde{X}$ be soft $T_{0}$ and soft $R_{1}$ and $x_{e}, y_{e^{\prime}} \in \tilde{X}$ such that $x_{e} \neq y_{e^{\prime}}$. Since $\tilde{X}$ is soft $T_{0}$, so by Definition 2.17 , there exists a soft open set $(G, A)$ 
containing one of the points but not the other. Suppose that $x_{e} \in(G, A)$ and $y_{e^{\prime}} \notin(G, A)$ implies that $(G, A) \sqcap\left\{y_{e^{\prime}}\right\}=\tilde{\phi}$, and then $x_{e} \notin \tilde{s} c l\left(\left\{y_{e^{\prime}}\right\}\right)$ this implies that $\tilde{s} c l\left(\left\{x_{e}\right\}\right) \neq \tilde{s} c l\left(\left\{y_{e^{\prime}}\right\}\right)$ and since $\tilde{X}$ is soft $R_{1}$, so there exist two disjoint soft open sets $(G, A)$ and $(H, A)$ such that $\tilde{s} c l\left(\left\{x_{e}\right\}\right) \sqsubseteq(G, A)$ and $\tilde{s} c l\left(\left\{y_{e^{\prime}}\right\}\right) \sqsubseteq(H, A)$ implies that $x_{e} \in(G, A)$ and $y_{e^{\prime}} \in(H, A)$. Thus $(X, \tau, A)$ is soft $T_{2}$.

\section{Functions with soft closed graphs}

In this section we introduce the concepts of the soft closed graph and the soft cluster set of a function $f_{p u}:(X, \tau, A) \rightarrow(Y, \mu, B)$ and give several related properties. Also, we proved that a function has a soft closed graph if and only if its soft cluster set at a fixed point degenerate.

Definition 4.1. The graph of a function $f_{p u}:(X, \tau, A) \rightarrow(Y, \mu, B)$ is denoted by $G\left(f_{p u}\right)$ and it is soft closed in $\tilde{X} \times \tilde{Y}$, if for each $\left(x_{e}, y_{e^{\prime}}\right) \in$ $G\left(f_{p u}\right)$, there exist two soft open sets $(U, A)$ and $(V, B)$ containing $x_{e}$ and $y_{e^{\prime}}$ respectively such that $(U, A) \times(V, B) \sqcap G\left(f_{p u}\right)=\tilde{\phi}$.

The following lemma follows from Definition 4.1.

Lemma 4.2. The function $f_{p u}:(X, \tau, A) \rightarrow(Y, \mu, B)$ has a soft closed graph if and only if for each $x_{e} \in \tilde{X}$ and $y_{e^{\prime}} \in \tilde{Y}$ such that $f_{p u}\left(x_{e}\right) \neq$ $y_{e^{\prime}}$, there exist two soft open sets $(U, A)$ and $(V, B)$ containing $x_{e}$ and $y_{e^{\prime}}$ respectively such that $f_{p u}((U, A) \sqcap(V, B))=\tilde{\phi}$.

Proposition 4.3. If $f_{p u}:(X, \tau, A) \rightarrow(Y, \mu, B)$ is an injective function with soft closed graph, then $\tilde{X}$ is a soft $T_{1}$ space.

Proof. Let $\left(x_{1}\right)_{e_{1}}$ and $\left(x_{2}\right)_{e_{2}}$ be two distinct points in $\tilde{X}$. Since $f_{p u}$ is injective, so $f_{p u}\left(\left(x_{1}\right)_{e_{1}}\right) \neq f_{p u}\left(\left(x_{2}\right)_{e_{2}}\right)$. Let $f_{p u}\left(\left(x_{1}\right)_{e_{1}}\right)=\left(y_{1}\right)_{e_{1}^{\prime}}$ thus $f_{p u}\left(\left(x_{2}\right)_{e_{2}}\right)=\left(y_{2}\right)_{e_{2}^{\prime}}$, by Lemma 4.2 , there exist two soft open sets $(U, A)$ and $(V, B)$ containing $\left(x_{2}\right)_{e_{2}}$ and $\left(y_{1}\right)_{e_{1}^{\prime}}$ respectively, such that $f_{p u}((U, A)) \sqcap$ $(V, B)=\tilde{\phi}$, then $(U, A) \sqcap f_{p u}^{-1}(V, B)=\tilde{\phi}$. We get $f_{p u}\left(\left(x_{1}\right)_{e_{1}}\right)=\left(y_{1}\right)_{e_{1}^{\prime}} \in$ $(V, B)$, then $\left(x_{1}\right)_{e_{1}} \in f_{p u}^{-1}(V, B)$ implies that, $\left(x_{1}\right)_{e_{1}} \notin(U, A)$. Again consider $f_{p u}\left(\left(x_{2}\right)_{e_{2}}\right)=\left(y_{2}\right)_{e_{2}^{\prime}}$ implies that $f_{p u}\left(\left(x_{1}\right)_{e_{1}}\right)=\left(y_{2}\right)_{e_{2}^{\prime}}$. Since the graph of $f_{p u}$ is soft closed, so there exist soft open sets $\left(U_{1}, A\right)$ containing $\left(x_{1}\right)_{e_{1}}$ and $\left(V_{1}, A\right)$ containing $\left(y_{2}\right)_{e_{2}^{\prime}}$ such that $f_{p u}\left(\left(U_{1}, A\right) \sqcap\left(V_{1}, A\right)\right)=\tilde{\phi}$, so $\left(U_{1}, A\right) \sqcap f_{p u}^{-1}\left(V_{1}, A\right)=\tilde{\phi}$, we obtain $f_{p u}\left(\left(x_{2}\right)_{e_{2}}\right)=\left(y_{2}\right)_{e_{2}^{\prime}} \in\left(V_{1}, A\right)$, so $\left(x_{2}\right)_{e_{2}} \in f_{p u}^{-1}\left(V_{1}, A\right)$ and hence $\left(x_{2}\right)_{e_{2}} \notin\left(U_{1}, A\right)$. Therefore, $\tilde{X}$ is a soft $T_{1}$ space. 
Proposition 4.4. If $f_{p u}:(X, \tau, A) \rightarrow(Y, \mu, B)$ is a surjective function with soft closed graph, then $\tilde{Y}$ is a soft $T_{1}$ space.

Proof. Let $\left(y_{1}\right)_{e_{1}^{\prime}}$ and $\left(y_{2}\right)_{e_{2}^{\prime}}$ be two distinct points in $\tilde{Y}$. Since $f_{p u}$ is surjective, so there exists a point $\left(x_{1}\right)_{e_{1}} \in \tilde{X}$, with $f_{p u}\left(\left(x_{1}\right)_{e_{1}}\right)=\left(y_{1}\right)_{e_{1}^{\prime}}$ then $f_{p u}\left(\left(x_{1}\right)_{e_{1}}\right) \neq\left(y_{2}\right)_{e_{2}^{\prime}}$. Therefore, $\left(\left(x_{1}\right)_{e_{1}},\left(y_{2}\right)_{e_{2}^{\prime}}\right) \notin G\left(f_{p u}\right)$, since the graph of $f_{p u}$ is soft closed, by Lemma 4.2, there exist two soft open sets $\left(U_{1}, A\right)$ containing $\left(x_{1}\right)_{e_{1}}$ and $\left(V_{2}, A\right)$ containing $\left(y_{2}\right)_{e_{2}^{\prime}}$ such that $f_{p u}\left(\left(U_{1}, A\right) \sqcap\right.$ $\left.\left(V_{2}, A\right)\right)=\tilde{\phi}$, We obtain $\left(y_{2}\right)_{e_{2}^{\prime}} \in\left(V_{2}, A\right)$, and $\left(x_{1}\right)_{e_{1}} \in\left(U_{1}, A\right)$ implies that $f_{p u}\left(\left(x_{1}\right)_{e_{1}}\right) \in f_{p u}\left(U_{1}, A\right)$, so $\left(y_{1}\right)_{e_{1}^{\prime}} \notin\left(V_{2}, A\right)$. Again from the surjectivity of $f_{p u}$ there exists $\left(x_{2}\right)_{e_{2}} \in \tilde{X}$ with $f_{p u}\left(\left(x_{2}\right)_{e_{2}}\right)=\left(y_{2}\right)_{e_{2}^{\prime}}$, then $f_{p u}\left(\left(x_{2}\right)_{e_{2}}\right) \neq\left(y_{1}\right)_{e_{1}^{\prime}}$, thus $\left(\left(x_{2}\right)_{e_{2}},\left(y_{1}\right)_{e_{1}^{\prime}}\right) \notin G\left(f_{p u}\right)$ and the graph of $f_{p u}$ is soft closed, there exist two soft open sets $\left(U_{2}, A\right)$ and $\left(V_{1}, A\right)$ containing $\left(x_{2}\right)_{e_{2}}$ and $\left(y_{1}\right)_{e_{1}^{\prime}}$ respectively, such that $f_{p u}\left(\left(U_{2}, A\right)\right) \sqcap\left(V_{1}, A\right)=\tilde{\phi}$. We get $\left(x_{2}\right)_{e_{2}} \in\left(U_{2}, A\right)$ implies that $\left(y_{2}\right)_{e_{2}^{\prime}}=f_{p u}\left(\left(x_{2}\right)_{e_{2}}\right) \in f_{p u}\left(\left(U_{2}, A\right)\right)$, so $\left(y_{2}\right)_{e_{2}^{\prime}} \notin\left(V_{1}, A\right)$. It follows that $\tilde{Y}$ is soft $T_{1}$.

The following corollary follows from Proposition 4.3 and Proposition 4.4 .

Corollary 4.5. If $f_{p u}:(X, \tau, A) \rightarrow(Y, \mu, B)$ is a bijective function with soft closed graph, then both $\tilde{X}$ and $\tilde{Y}$ are soft $T_{1}$ spaces.

Proposition 4.6. If $f_{p u}:(X, \tau, A) \rightarrow(Y, \mu, B)$ is is soft continuous and $\tilde{Y}$ is a soft $T_{2}$ space, then $G\left(f_{p u}\right)$ is soft closed.

Proof. Let $\left(x_{e}, y_{e^{\prime}}\right) \notin G\left(f_{p u}\right)$. Then $f_{p u}\left(x_{e}\right) \neq y_{e^{\prime}}$ and since $\tilde{Y}$ is a soft $T_{2}$ space, there exist soft open sets $(U, A)$ and $(V, B)$ such that $f_{p u}\left(x_{e}\right) \in(U, A), y_{e^{\prime}} \in(V, B)$ and $((U, A) \sqcap(V, B))=\tilde{\phi}$. Since $f_{p u}$ is soft continuous, so there exists a soft open set $(G, A)$ containing $x_{e}$ such that $f_{p u}(G, A) \sqsubseteq(U, A)$. Hence, we have $f_{p u}((G, A)) \sqcap(V, B)=\tilde{\phi}$. Therefore, by Lemma $4.2, G\left(f_{p u}\right)$ is soft closed.

Definition 4.7. Let $f_{p u}:(X, \tau, A) \rightarrow(Y, \mu, B)$ be any soft function. The soft cluster set of $f_{p u}$ at $x_{e}$ is denoted by $\tilde{s} C\left(f_{p u}, x_{e}\right)$ is the set of all points $y_{e^{\prime}} \in \tilde{Y}$ such that whenever there exists a filter base $\mathcal{F}$ soft converges to the point $x_{e}$, the filter base $\left\langle f_{p u}(\mathcal{F})>\right.$ soft converges to the point $y_{e^{\prime}}$.

The following theorem is a characterization of soft cluster set of a function $f_{p u}$. 
Theorem 4.8. Let $f_{p u}:(X, \tau, A) \rightarrow(Y, \mu, B)$ be any function and $x_{e} \in \tilde{X}$. Then the following statements are equivalent:

1. $y_{e^{\prime}} \in \tilde{s} C\left(f_{p u}, x_{e}\right)$,

2. $y_{e^{\prime}} \in \sqcap\left\{\tilde{s} c l\left(f_{p u}((U, A)): \forall \quad(U, A) \in \tilde{s} N\left(x_{e}\right)\right\}\right.$,

3. $f_{p u}\left(\tilde{s} N\left(x_{e}\right)\right)$ is soft accumulates to $y_{e^{\prime}}$,

4. $f_{p u}^{-1}\left(\tilde{s} N\left(y_{e^{\prime}}\right)\right)$ is soft accumulates to $x_{e}$,

5. $x_{e} \in \sqcap\left\{\tilde{s} c l\left(f_{p u}^{-1}((V, B)): \forall \quad(V, B) \in \tilde{s} N\left(y_{e^{\prime}}\right)\right\}\right.$,

Proof. $\quad(1) \Rightarrow(2)$. Let $y_{e^{\prime}} \in \tilde{s} C\left(f_{p u}, x_{e}\right)$, so there exists a filter base $\mathcal{F}$ soft converges to the point $x_{e}$ and $\left\langle f_{p u}(\mathcal{F})>\right.$ soft converges to the point $y_{e^{\prime}}$. Suppose that $(U, A)$ is any soft open set containing $x_{e}$, since $\mathcal{F}$ soft converges to $x_{e}$, so $(U, A) \in \mathcal{F}$ and $\left\langle f_{p u}(\mathcal{F})\right\rangle$ soft converges to the point $y_{e^{\prime}}$. Therefore, $y_{e^{\prime}} \in \tilde{s} c l\left(f_{p u}\left(x_{e}\right)\right)$ implies that $y_{e^{\prime}} \in \tilde{s} c l\left(f_{p u}((U, A))\right.$ for each soft open set $(U, A)$ containing $x_{e}$. Hence $y_{e^{\prime}} \in \sqcap\left\{\tilde{s} c l\left(f_{p u}((U, A))\right.\right.$ : $\left.\forall \quad(U, A) \in \tilde{s} N\left(x_{e}\right)\right\}$.

$(2) \Rightarrow(3)$. Let $y_{e^{\prime}} \in \sqcap\left\{\tilde{s} c l\left(f_{p u}((U, A)): \forall \quad(U, A) \in \tilde{s} N\left(x_{e}\right)\right.\right.$, so $y_{e^{\prime}} \in$ $\tilde{s} c l\left(f_{p u}((U, A))\right.$ for each soft open set $(U, A)$ containing $x_{e}$. Then $f_{p u}((U, A)) \sqcap$ $(V, B) \neq \tilde{\phi}$ for each soft open sets $(U, A)$ containing $x_{e}$ and $(V, B)$ containing $y_{e^{\prime}}$ implies that $f_{p u}\left(\tilde{s} N\left(x_{e}\right) \sqcap(V, B) \neq \tilde{\phi}\right.$ for every soft open set $(V, B)$ containing $y_{e^{\prime}}$ and hence $f_{p u}\left(\tilde{s} N\left(x_{e}\right)\right)$ is soft accumulates to $y_{e^{\prime}}$.

$(3) \Rightarrow(4)$. Let $f_{p u}\left(\tilde{s} N\left(x_{e}\right)\right)$ is soft accumulates to $y_{e^{\prime}}$, which implies that $f_{p u}\left(\tilde{s} N\left(x_{e}\right) \sqcap(V, B) \neq \tilde{\phi}\right.$ for each $(V, B) \in \tilde{s} N\left(y_{e^{\prime}}\right)$, thus $(U, A) \sqcap f_{p u}^{-1}\left(\tilde{s} N\left(y_{e^{\prime}}\right)\right) \neq$ $\tilde{\phi}$ for every soft open set $(U, A)$ in $\tilde{X}$ containing $x_{e}$ it follows that $f_{p u}^{-1}\left(\tilde{s} N\left(y_{e^{\prime}}\right)\right)$ is soft accumulates to $x_{e}$.

$(4) \Rightarrow(5)$. Assume that $f_{p u}^{-1}\left(\tilde{s} N\left(y_{e^{\prime}}\right)\right)$ is soft accumulates to $x_{e}$, so $(U, A) \sqcap$ $f_{p u}^{-1}\left(\tilde{s} N\left(y_{e^{\prime}}\right)\right) \neq \tilde{\phi}$, for every soft open set $(U, A)$ containing $x_{e}$. It follows that $(U, A) \sqcap f_{p u}^{-1}((V, B)) \neq \tilde{\phi}$ for every soft open set $(U, A)$ in $\tilde{X}$ containing $x_{e}$ and $(V, B)$ in $\tilde{Y}$ containing $y_{e^{\prime}}$. Hence, $x_{e} \in \tilde{s} c l\left(f_{p u}^{-1}((V, B))\right)$ for every soft open set $(V, B)$ containing $y_{e^{\prime}}$. This shows that $x_{e} \in \sqcap\left\{\tilde{s} c l\left(f_{p u}^{-1}((V, B))\right.\right.$ : $\left.\forall \quad(V, B) \in \tilde{s} N\left(y_{e^{\prime}}\right)\right\}$.

$(5) \Rightarrow(1)$. Since $S O\left(\tilde{X}, x_{e}\right)$ is a filter base which is soft converges to the point $x_{e}$, then $S O\left(\tilde{X}, x_{e}\right)$ is contained in an ultra filter $\mathcal{F}$ on $\tilde{X}$ which is also soft converges to $x_{e}$, so there exists $(F, A) \in \mathcal{F}$ such that $(F, A) \sqsubseteq(U, A)$ for every soft open set $(U, A)$ in $\tilde{X}$ containing $x_{e}$, so $(U, A) \in \mathcal{F}$. By $(5), x_{e} \in \tilde{s} c l\left(f_{p u}^{-1}((V, B))\right.$ for every soft open set $(V, B)$ containing $y_{e^{\prime}}$. So 
$(U, A) \sqcap f_{p u}^{-1}((V, B)) \neq \tilde{\phi}$, implies that $f_{p u}((U, A)) \sqcap(V, B) \neq \tilde{\phi}$ for every soft open set $(U, A)$ containing $x_{e}$ and $(V, B)$ containing $y_{e^{\prime}}$. Hence, by Proposition 2.16, $f_{p u}(\mathcal{F})$ is an ultra filter base which is soft accumulates to the soft point $y_{e^{\prime}}$,so by Theorem $2.15, f_{p u}(\mathcal{F})$ is soft convergent to $y_{e^{\prime}}$.

By using the concept of soft cluster set of a function $f_{p u}:(X, \tau, A) \rightarrow$ $(Y, \mu, B)$ we obtain some properties and characterizations of the soft graph of the function $f_{p u}$. We start by the following result which is a relation between a function with soft closed graph and soft cluster set of the function. First we introduce the concept of degenerate soft cluster set.

Definition 4.9. Let $(X, \tau, A)$ be a soft topological space, the degenerate soft cluster set of $f_{p u}$ is a soft cluster set which contains exactly one element.

Theorem 4.10. Let $f_{p u}:(X, \tau, A) \rightarrow(Y, \mu, B)$ be any function, the graph of $f_{p u}$ is soft closed if and only if the soft cluster set of $f_{p u}$ at $x_{e}$ is degenerate.

Proof. Let $y_{e^{\prime}}$ be any point in $\tilde{Y}$ different from $f_{p u}\left(x_{e}\right)$. By Lemma 4.2 , there exist $(U, A) \in S O\left(\tilde{X}, x_{e}\right)$ and $(V, B) \in S O\left(\tilde{Y}, y_{e^{\prime}}\right)$ such that $f_{p u}((U, A)) \sqcap(V, B) \neq \tilde{\phi}$. This implies that $y_{e^{\prime}} \notin \tilde{s} c l\left(f_{p u}((U, A))\right.$ and by Theorem 4.8(2), $y_{e^{\prime}} \in \tilde{s} C\left(f_{p u}, x_{e}\right)$. Hence $\tilde{s} C\left(f_{p u}, x_{e}\right)=\left\{f_{p u}\left(x_{e}\right)\right\}$.

Conversely, suppose $G\left(f_{p u}\right)$ is not soft closed. This implies that there exists $\left(x_{e}, y_{e^{\prime}}\right) \notin G\left(f_{p u}\right)$ such that $f_{p u}((U, A)) \sqcap(V, B) \neq \tilde{\phi}$ for every soft open set $(U, A)$ in $\tilde{X}$ containing $x_{e}$ and $(V, B)$ in $\tilde{Y}$ containing $y_{e^{\prime}}$, then $y_{e^{\prime}} \in$ $\tilde{s} c l\left(f_{p u}((U, A))\right.$ for each soft open set $(U, A)$ containing $x_{e}$ by Theorem 4.8(2), $y_{e^{\prime}} \in \tilde{s} C\left(f_{p u}, x_{e}\right)$ which contradicts the fact that $\tilde{s} C\left(f_{p u}, x_{e}\right)=$ $\left\{f_{p u}\left(x_{e}\right)\right\}$. Therefore, $G\left(f_{p u}\right)$ is soft closed.

The following results follows from Theorem 4.10 and the definition of soft cluster set of a function.

Corollary 4.11. The function $f_{p u}:(X, \tau, A) \rightarrow(Y, \mu, B)$ has a soft closed graph if and only if there exists a filter base $\mathcal{F}$ soft converges to a point $x_{e}$, the filter base $f_{p u}(\mathcal{F})$ soft converges to a point $y_{e^{\prime}}$ and $y_{e^{\prime}}=f_{p u}\left(x_{e}\right)$..

Corollary 4.12. Let $f_{p u}:(X, \tau, A) \rightarrow(Y, \mu, B)$ be any function. The graph of $f_{p u}$ is soft closed if and only if

$$
f_{p u}\left(x_{e}\right) \in \sqcap\left\{\tilde{s} c l\left(f_{p u}((U, A)): \forall \quad(U, A) \in \tilde{s} N\left(x_{e}\right)\right\}\right.
$$




\section{Conclusion}

In the last two decades the soft set theory, new definitions, examples, new classes of soft sets, and properties for mappings between different classes of soft sets are introduced and studied. After then, the theory of soft topological spaces is investigated. This paper continues the study of the theory of soft topological spaces. In section 3, we present the notion of soft $R_{i}$ spaces for $i=0,1$, we get several characterizations and properties of these two spaces. In section 4, we obtain nice results concerning functions with soft closed graphs and its relations with the notion of soft convergence and cluster set of a function.

\section{References}

[1] M. Akdag, and A. Ozkan, "On soft preopen sets and soft pre separation axioms", Gazi university journal of science, vol. 27, no. 4, pp. 1077-1083, 2014.

[2] S. Bayramov and and C. G. Aras, "A new approach to separability and compactness in soft topological spaces", Turkic World Mathematical Society Journal of pure and applied mathematics, vol. 9, no. 1, pp. 82-93, 2018.

[3] S. Hussain and B. Ahmad, "Some properties of soft topological spaces", Computers and mathematics with applications, vol. 62, no. 11, pp. 4058-4067, 2011. doi: 10.1016/ j.camwa.2011.09.051

[4] S. Hussain, "Soft separation axioms in soft topological spaces", Hacettepe journal of mathematics and statistics, vol. 44, no. 3, pp. 559-568, 2015.

[5] A. Kharal and B. Ahmad, "Mappings on soft classes", New mathematics and natural computation, vol. 7, no. 3, pp. 471-481, 2011. doi: 10.1142/ S1793005711002025

[6] P. K. Maji, R. Biswas, and A. R. Roy, "Soft set theory", Computers and mathematics with applications, vol. 45, no. 4-5, pp. 555-562, 2003. doi: 10.1016/ S0898-1221(03)00016-6

[7] D. Molodtsov, "Soft set theory-first results", Computers and mathematics with applications, vol. 37, no. 4-5, pp. 19-31, 1999. doi: 10.1016/ S0898-1221(99)00056-5 
[8] R. Sahin and A. Kucuk, "Soft filters and their convergence properties", Annals of fuzzy mathematics and informatics, vol. 6, no. 3, pp. 529-543, 2013.

[9] M. Shabir and M. Naz, "On soft topological spaces", Computers and mathematics with applications, vol. 61, no. 7, pp. 1786-1799, 2011. doi: 10.1016/ j.camwa.2011.02.006

[10] M. E. El-Shafei, M. Abo-Elhamayel, and T. M. Al-Shami, "Partial soft separation axioms and soft compact spaces", Filomat, vol. 32, no. 13, pp. 4755-4771, 2018. doi: 10.2298/ FIL1813755E

[11] P. Wang and J. He, "Characterization of soft compact spaces based on soft filter", Journal of theoretical and applied information technology, vol. 79, no. 3, pp. 431-436, 2015.

[12] Ş. Yüksel, N. Tozlu, and Z. G. Ergül, "Soft filter", Mathematical sciences, vol. 8, no. 1, 2014. doi: 10.1007/ s40096-014-0119-4

[13] I. Zorlutuna, M. Akdag, W. K. Min and S. Atmaca, Remarks on soft topological spaces, Annals of fuzzy mathematics and informatics, vol. 3, no. 2, pp. 171-185, 2012.

[14] İ. Zorlutuna and H. Çakır, "On continuity of soft mappings", Applied mathematics and information sciences, vol. 9, no. 1, pp. 403-409, 2015. doi: 10.12785/amis/ 090147 
Alias B. Khalaf

Department of Mathematics,

College of Science,

University of Duhok,

Kurdistan Region

Iraq

e-mail: aliasbkhalaf@uod.ac

Corresponding author

Nehmat K. Ahmed

Department of Mathematics,

College of Education,

Salahaddin University,

Kurdistan-Region,

Iraq

e-mail: nehmat.ahmed@su.edu.krd

and

Qumri H. Hamko

Department of Mathematics,

College of Education,

Salahaddin University,

Kurdistan-Region,

Iraq

e-mail: qumri.hamko@su.edu.krd 